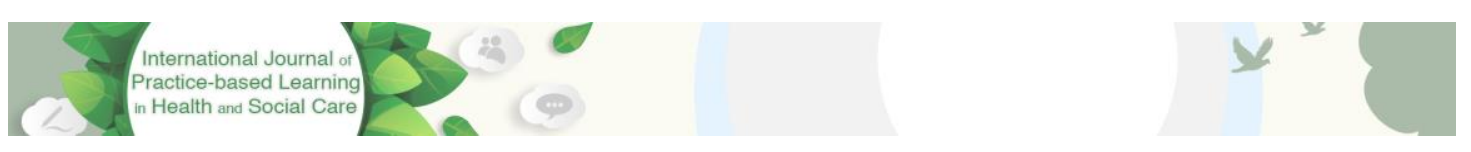

International Journal of Practice-based Learning in Health and Social Care Vol. 8 No 2 December 2020, pages 57-67

\title{
Supporting Newly Qualified Diagnostic Radiographers: Are We Getting It Right?
}

\author{
*Jane Harvey-Lloyd $d^{a}$ and Jane Morris ${ }^{b}$ \\ ${ }^{a}$ School of Health Sciences, University of Suffolk, UK; ${ }^{b}$ University of Brighton, UK
}

\begin{abstract}
The NHS is facing a rising demand in services and consequently, newly qualified practitioners are required to possess a much wider set of skills than ever before. In diagnostic radiography, this pressure is underpinned by the expanding role that diagnostic imaging now plays in many patient pathways. Despite the need for structured support for graduates which has been acknowledged by a range of studies and the introduction of the Preceptorship Framework for Newly Registered Nurses, Midwives and Allied Health Professionals (Department of Health $[\mathrm{DoH}], 2010)$ the uptake in radiography is limited. A longitudinal study employed an interpretive phenomenological methodology collating data from nine participants during their first year as band five radiographers. Each participant was interviewed at three, six and twelve months. Six main themes were identified during a wider PhD study Being and Becoming a Diagnostic Radiographer (Harvey-Lloyd, 2018). This article explores one of the key sub themes 'structured support' which strongly featured in the three- and six-month interviews. Two of the nine participants received a structured support programme during their first 12 months of transition as newly qualified radiographers. These programmes focussed on the completion of tasks and were mechanistic in approach. This type of support ignores the process of transformation into the role of a diagnostic radiographer and fails to adequately support those from Generation Z. A model of preceptorship is proposed which combines skills and competence development, supporting transition and personal and professional development; thus, providing the newly qualified radiographers with a holistic range of support.
\end{abstract}

Keywords: newly qualified; phenomenology; preceptorship; radiographer; transition

\section{Introduction}

The pressures facing the National Health Service (NHS) are increasing due to the rising demand for services and the current economic, social and political influences (Harvey-Lloyd et al., 2019; NHS Confederation, 2014). The service changes that are now required to manage such challenges require staff to possess a much wider set of skills than ever before and consistently demand resilience and commitment from health care practitioners (Richez, 2014; McAllister \& McKinnon, 2009). This environment exacerbates the pressure now placed on new graduates to fit in and learn quickly as they navigate the

*Corresponding Author: Dr Jane M Harvey-Lloyd, PhD MSc DCR(R) PGCE DLC CTC RPS, Associate Professor, School of Health and Sports Sciences, University of Suffolk, UK Email: j.harvey-1loyd@uos.ac.uk

Journal URL: https://publications.coventry.ac.uk/index.php/pblh

Harvey-Lloyd, J., \& Morris, J. (2020). Supporting newly qualified diagnostic radiographers: Are we getting it right? International Journal of Practice-based Learning in Health and Social Care, 8(2), 57-67. DOI 10.18552/ijpblhsc.v8i2.673

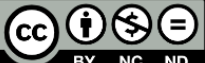

(C) 2020 Jane Harvey-Lloyd, \& Jane Morris. This Open Access article is distributed under the terms of the Creative Commons Attribution Attribution-Non-Commercial No Derivatives 4.0 International License (https://creativecommons.org/licenses/by-nc-nd/4.0/ ), which permits unrestricted non-commercial use, distribution, and reproduction in any medium, provided the original work is properly cited and is unaltered. 
territory of a newly qualified practitioner. Specifically, in diagnostic radiography this pressure is intensified by the increasing role that diagnostic imaging has in many patient care pathways, resulting in increasing numbers of procedures being undertaken and the complex management of patients with longterm conditions and co-morbidities (Harvey-Lloyd et al., 2019).

This article shares data from a $\mathrm{PhD}$ study which explored the process of being and becoming a diagnostic radiographer in which needing support emerged as a key theme (see Harvey-Lloyd et al., 2019). The key themes identified as part of this study are Needing Support, Settling In, Developing Confidence, Becoming Established, Feeling Useful and Looking Forward.

There were four sub-themes relating to the main theme of needing support: Reality hits, Structured support, Support from colleagues and Peer support. The evidence from this study clearly identified the need for support for newly qualified practitioners and the data collated as part of the subtheme 'structured support' are used to underpin the discussion and focus of this article (Harvey-Lloyd et al., 2019).

\section{Literature review}

In 2010, the Department of Health (DoH) introduced a Preceptorship Framework for Newly Registered Nurses, Midwives and Allied Health Professionals in the National Health Service (Department of Health, $(\mathrm{DoH}), 2010)$. The intention of the framework was to ensure that newly qualified health professionals received the support necessary to give them the best start possible in their professional role. It focused on newly qualified health professionals having protected time and expert support to help them apply their academic knowledge and practice placement experiences in real life situations as accountable professionals. Some years ago, a preceptorship scheme was proposed by the Association of Radiography Educators on behalf of the Society of Radiographers (SoR) Harvey-Lloyd (2018). This was introduced as a way of supporting newly qualified radiographers during their transition period. Anecdotal evidence and discussions at various national committees suggested that the uptake of preceptorship schemes in diagnostic radiography in departments across the United Kingdom (UK) was small. However, in departments where it was offered, it was connected to the progression of the link grade from a band five to band six radiographer where at the end of the preceptorship period, the radiographer was able to apply for regrading to a band six post. This originally facilitated promotion from a first post to a more senior role within 24 months. However, with the introduction of Agenda for Change, this opportunity was removed and with it many preceptorship schemes diminished. Those that have continued to provide preceptorship often use the SoR model or its updated version (both undated) which is predominantly product-driven rather than process-driven and rather mechanistic in approach, focussing on twelve activities which the preceptee needs to complete. These activities are:

- Familiarise with CPD Now (the online Continuing Professional Development system hosted by the SCoR) contents and operation

- Evaluate technical aspects of your practice

- Evaluate interpersonal and communication skills

- Evaluate knowledge of department procedures, protocols etc.

- Implication of SCoR policy on consent

- Review an article in Synergy (editorial board reviewed)

- Review an article in Radiography (peer reviewed journal)

- Undertake CoR e-learning module

- Undertake self-directed learning

- Attend departmental or in-house CPD

- Attend conference/study day/seminar etc.

- Study a department policy or procedure re: health and safety

The current model is without doubt limiting in terms of professional development and is heavily linked to outcomes. Moving to a process model would be much more appropriate given the different values, perceptions, expectations and motivations of Generation $\mathrm{Z}$ who need a more-tailored, individualised support system than those from previous generations (Jones et al., 2015). However, despite the 
introduction of the Preceptorship Framework and the differing needs of the future generation, radiography as a profession does not yet seem to have embraced this approach ( $\mathrm{DoH}, 2010$; Jones et al., 2015).

Preceptorship is defined as "a period of structured transition for the newly registered practitioner during which s/he will be supported by a preceptor, to develop their confidence as an autonomous professional, refine skills, values and behaviours and to continue on their journey of life-long learning" (DoH, 2010, p. 11). The literature suggests that this will underpin both professional and personal development during this time as they are becoming a diagnostic radiographer (Black et al., 2010; Boychuk Duchscher, 2008; Fenwick et al., 2012; Gerrish, 1990; MacKay et al., 2008; Mooney, 2007; Tryssenaar \& Perkins, 2001). Role transition itself has been widely investigated by a range of healthcare professions but as yet there does not seem to be a firm consensus on type, depth and length of support needed It has been seen across the healthcare professions that the support given is both variable and inconsistent (Black et al., 2010; Boychuk Duchscher, 2008; Fenwick et al., 2012; Gerrish, 1990; MacKay et al., 2008; Mooney, 2007; Tryssenaar \& Perkins, 2001).

The need for a structured learning experience for new graduates was highlighted by Black et al. (2010) in order to support the transition from student to therapist. They identified how little is known in terms of workplace learning during this time and recognised the multidimensional complexity of competence and expertise. In investigating the learning and development of a promising novice, it was found that the emergence of professional identity and role transition is central to this process and closely linked to growing confidence. The concept model proposed by Black et al. (2010) as a result of their study of the experience of newly qualified OTs and PTs, focussed on four key concepts related to the transition period: clinical environment and practice community, learning through experience, growing confidence and professional identity formation and role transition.

In response to the challenges faced by newly qualified midwives and nurses during the transition period, a national development programme known as the Flying Start NHS was introduced in Scotland in 2006 (NHS Education for Scotland, 2006). The programme consists of ten units and is a web-based or CDROM programme which aims to develop the confidence and competence of newly qualified nurses and midwives during their first year of practice. The units are Communication, Clinical skills, Teamwork, Safe practice, Research for practice, Equality and diversity, Policy, Reflective practice, Professional development and Career pathways. The programme allows practitioners to map their progress against the Knowledge and Skills Framework (KSF), supporting professional and personal development. An evaluation of this programme was undertaken to establish the impact of the programme on the confidence, competence and career development of the newly qualified practitioners (Banks et al., 2011). In total 547 newly qualified practitioners completed the survey comprising of 334 nurses, 20 midwives and 193 Allied Health Professions (AHPs). Of the total respondents, 15 were diagnostic radiographers (2.7\%) and accounted for $7.8 \%$ of the AHP section. It was evident from the study that the newly qualified practitioners were still facing several challenges during their transition. Although approximately $75 \%$ of respondents were allocated a mentor to provide support whilst undertaking the Flying Start NHS programme, they waited between 4-9 weeks for allocation with AHPs waiting longer on average than nurses and midwives. It was noted that the satisfaction with the support received from mentors was mixed with a higher proportion of midwives stating that the support received from their mentors was good or very good. Support from mentors in the community was also more positively rated than in the acute setting. There were no separate figures given for the AHPs due to small numbers.

Overall, the study concluded that the majority of newly qualified practitioners taking part in the study found completing the programme helpful in terms of developing confidence and clinical skills. The most useful learning units were learning the job, becoming a member of the team and orientation/induction to the clinical area. Many of the respondents however, reported difficulties in completing the programme due to time constraints and a lack of support/partnership. This is a concern if the programme is to fulfil the aim of improving recruitment and retention. Unfortunately, the number of AHPs and specifically, diagnostic radiographers, that took part in this project was small. However, overall, the programme was positively evaluated and could be considered as a support mechanism for new graduates in England in the future. 
The issue of supporting newly qualified radiographers was explored by Naylor et al. (2016) and two support mechanisms were discussed: peer support and scaffolding. It was found that peer support was valued by all participants whether formal or informal. Although there is limited literature on peer support during transition for AHPs, this study established that peer support reduced anxiety with regards to starting work; provided a personal support group; and reduced isolation as it encouraged a sense of belonging. It was also was recognised in this study that some departments offered structured support aligned to that which was initially introduced by Bruner (Fleer 1990). This is where support is reduced over time as the graduate gains experience, develops their practice and increases in confidence. The rate at which support is decreased is dependent of the development of the individual and is therefore personcentred (Cole et al., 1978).

The work of Dall'Alba (2009), on professional ways of being and becoming, challenges the epistemological aspect of education and in this case the transition period. She argues that if consideration was given to the ontological aspect of transition then 'being and becoming' would encompass the transformation into a professional (Dall'Alba and Barnett, 2007). This involves not only the element of performance but more importantly the possibilities to learn and grow which are enhanced by reflection, interaction with others and being open to new ways of working. This is also discussed by Barnett (2009) who recognises the need for students to develop transferable skills. He argues that learning to learn is an essential graduate skill, proposing that knowledge no longer holds the power it once did.

Harvey-Lloyd et al. (2012) identified in their study the transition from newly qualified radiographer to practitioner remains a stressful period, especially in the first six months. Eight of the nine participants experienced 'reality shock' and this was exacerbated by their lack of experience at dealing with an unfamiliar work environment. The issue of structured support was highlighted as an important subtheme by the authors and this forms the basis for this article.

\section{Methodology}

In using a longitudinal, qualitative prospective approach (Cohen et al., 2000), this study explored the experience of transition from student to practitioner in diagnostic radiography specifically focusing on being and becoming a radiographer. Interpretive phenomenology was chosen as the suitable methodology for this study since it focuses on human experience and the subjective experience of a phenomenon (Kafle, 2011). It sits firmly in the domain of qualitative research and is underpinned by the subjective paradigm of ontology and in the interpretivist paradigm of epistemology; the underlying philosophy being that not everything can be explained by a scientific method and that there is no such thing as an objective truth (Wiercinski, 2009). Based within the humanistic paradigm and embedded in qualitative research, its purpose is to describe the first-hand experience of those involved with the phenomenon (Cresswell, 2007). In this case, it explored newly qualified radiographers' perceptions of the world of diagnostic radiography and its personal meaning to each of them (Langdridge, 2007). A total of nine participants were recruited, using a combination of purposive and criterion sampling (Cresswell, 2007). These participants were a gender mix of 5:4 ratio male to female and worked in seven diagnostic imaging departments in the UK. The participant inclusion and exclusion criteria are listed in Table 1. The participants were recruited at the end of their degree programme by a Gatekeeper (a nursing lecturer) and then the data was collated during their first year in practice.

Using this approach allowed the collation of rich and meaningful data. This was supported by the researcher's prior knowledge and experience of diagnostic radiography which enabled them to take an insider's perspective (Pringle et al., 2011) as they got to know the participants during the study.

Ethical approval was gained from the University Ethics Committee at the University where the PhD was undertaken and from the host university from which the participants were selected. 
Table 1: Participant Inclusion/Exclusion Criteria

\begin{tabular}{|l|l|}
\hline Inclusion criteria & Exclusion criteria \\
\hline $\begin{array}{l}\text { Students who graduated with a BSc (Hons) } \\
\text { Diagnostic Radiography degree from the HEI in } \\
\text { June 2013 }\end{array}$ & $\begin{array}{l}\text { Students gain employment in a NHS Hospital } \\
\text { Trust for a period of less than 1 year }\end{array}$ \\
$\begin{array}{l}\text { Students who gain employment in a NHS } \\
\text { Hospital Trust that was not their placement site } \\
\text { during their degree course }\end{array}$ & \\
\hline
\end{tabular}

Interviews allowed the researcher to collect data which provided insight into the participants' understanding and experiences of the phenomenon. Three interviews were undertaken with each participant at three-, six- and twelve-months post qualification. The three- and six-month time intervals were identified as critical times within the literature (Decker, 2009; Smith \& Pilling, 2007) who noted this was a very emotional and anxious time for newly qualified radiographers. Twenty-seven semi-structured interviews were undertaken, and all were audio recorded. In order to maximise the effectiveness of the interviews, two associated data collection tools were used at two different time intervals in order to complement and inform the semi-structured interviews. These were a Peaks and Troughs graph adapted by Harvey-Lloyd (2018) and a theme board. The purpose of the Peaks and Trough graph was to enable each participant to plot their experience of certain events on a graph and indicate the feelings associated with that experience. The participants of this study were asked to complete a Peaks and Troughs graph every day for each week during their first three months in post, thus encapsulating their early experiences. These were then brought to the first interview and used as an aide memoire. They were then explored in depth so that their feelings and emotions during the first three months were fully captured. The theme board was used as a visual reminder for the participants, using images to represent experiences and feelings. It is a powerful tool that can be used to convey messages behind the images and can be effectively utilised as a permanent record of a journey on which to reflect as well as a visual reminder of future goals and aspirations (Bligh, 1992). In this way data was accessed that may be missed by a traditional qualitative interview. By referring to the theme board during the final interview, the images triggered memories of and feelings about experiences (Leavy, 2009), that may otherwise have been forgotten. This led to the final interview being completely participant-led, guided by their reflections illustrated through the theme board.

The thematic analysis framework devised by Braun and Clarke (2006) was used for each interview in order to identify main and subthemes. The themes were then compared across the participants' experiences to allow comparisons to be made and a thematic map was developed. The thematic map provided an overview of the data and allowed examination of how the codes and themes were defined and named. The transcripts were then re-read, allowing an in-depth analysis of each individual experience but also allowed common themes to be drawn from the data, allowing insight into the phenomenon being explored.

\section{Findings and discussion}

The main theme of needing support featured strongly in the three-month interviews and was reflected on in many of the theme boards at the twelve-month stage. It consists of four sub-themes: Reality hits; Structured support; Support from colleagues; and Peer support. Reality hits was discussed in an earlier article to contextualise the environment in which newly qualified radiographers are entering. The subtheme, Structured support is further explored in this article with the use of pertinent quotations woven throughout the discussion. There are quotations presented from four of the participants to avoid repetition 
due to data saturation, however it should be noted that all participants discussed this in detail during their interviews.

\section{Structured support}

It was clear that being supported was an important aspect of the transition period for James, particularly when struggling with learning new ways of working. Throughout his interview he spoke about the lack of support he had received as a new graduate and how he had been disappointed by this. He had found it difficult to find his way at times. He felt that there was no one to turn to for support and that the only way forward was to just get on with it:

For me that was fine because that's what I was used to... and I enjoy kind of working on my own but I think it's a bit of a struggle sometimes when you're getting used to new protocols and there's not as much support as you sometimes think there would be.

(James, 3 months)

James revisited how he had felt during this time during his twelve-month interview:

.... as a newly qualified you don't exactly get supervised throughout the year [...] there's no kind of regular time to talk things through like that so you kind of have to bury it and get on with it....

(James, 12 months)

What James seems to be saying here is that it would have been useful to have the opportunity to regularly reflect on his practice with an experienced member of the team. He felt that because he was not given the chance to do this that he just had to keep on going and coping which may have resulted in him missing valuable learning opportunities.

It is evident that James had been given very little support with regard to learning by experience; one of the concepts introduced by Black et al. (2010). An opportunity to reflect with a more experienced practitioner as part of a preceptorship scheme would have ensured that James not only learned from his experiences but was also given the appropriate support to underpin his professional development, helping him to cultivate his clinical reasoning skills. This ties in well with the work by Dall'Alba (2009) who believes that giving an individual opportunities to grow and learn, underpin successful professional transition.

As the interviews progressed it became apparent that the participants' experience of any formal support mechanisms was indeed very different. Gareth continued to acknowledge the excellent support he felt that he had been given and went on to explain the preceptorship that had been put in place for him at his hospital.

So we started, and the introduction was really good... we were given all of our information for our preceptorship, [...] it's a six-month preceptorship which was clearly laid out, which included all the normal tick-lists, plus an audit as part of it and that was really daunting at the start to get so much information, and so much to fill out... but it was okay.

(Gareth, 3 months)

Gareth acknowledged that he felt completely overwhelmed by the amount of information he was given. Although it was well laid out and organised there seemed to be a lot required of him as part of this preceptorship and by his own admission, he found the expectations of this very daunting. It seemed that much of the preceptorship focused on paper-based exercises and was task-focussed. He was however, given support over the first six months and he undoubtedly viewed this a positive thing.

In complete contrast Ruby appeared to have received no structured support:

No, no reviews, no nothing. I've been told I'm getting a three-month review, whatever... which had not been discussed, I don't even think they know how long I've been here now.... They don't ask me how I'm getting on, there's an Excel sheet that you've got to tick when you're competent in something, so you just say 'yes' to all these different features of all the different machines, and that's all they're interested in me doing and as soon as that's all ticked off, they're happy.

(Ruby, 3 months) 
Ruby felt that she needed support, especially to help her fit into to a new department. It appeared that the department were satisfied as long as she undertook her job and kept imaging patients.

When discussing her experience there seemed to be a sense of disappointment and in some instance's disbelief from Ruby. She was unsure as to what to expect in terms of support and felt as though the department had little idea of how long she had been working there and how well she was performing. As with some of the other participants, she has been required to prove certain competencies, but this was simply a tick box activity. Ruby did not appear to have had any formal or informal feedback on how she was getting on and her assumption was that as long as the competencies she had been given were met, then that was all that was needed from her. This left Ruby feeling much more like a number in the workplace rather than a person. Again, there was a focus on task-based activities and little on individualised support and personal development. Consequently, Ruby felt despondent and disillusioned in these early months, feeling of little value.

Ruby revisited this at her twelve-month interview:

I kind of didn't feel that I had the appropriate support from work management just sort of telling me not helping you, you know not really any help there at all...but really just a bit of reassurance a bit more guidance would've just helped me to actually you know sort of fit into the department and feel reassured that what I was doing was right because at the time I just thought you know I don't if what I'm doing is right I'm just going with the flow and I'm just X-raying patients...

(Ruby, 12 months)

Later, Ruby referred to this early period as feeling like 'a ticking time bomb'. She explained how this feeling changed during the twelve months from 'a ticking time bomb on her shoulder' at six months to about nine months when it felt like the ticking time bomb disappeared. At the nine months' time period Ruby felt that she had finally settled into the department and was confident in her knowledge and abilities.

Adam, like Gareth was also given an induction period, albeit for the shorter period of three months. However, this differed in that it was not a preceptorship and was more akin to Ruby's experience which was focussed on training and competencies in specific areas. Adam was pleased with the in-depth training he received although does mention the length of time that it took.

... as time has gone on I've been trained in loads of different areas. The induction programme at ... is really elongated. Like I, started off in generals', then we went to theatres. And we had a good five, three-week blocks in each area. So, it's a really thorough training period and I'm still in my induction period, even three months in.

(Adam, 3 months)

Overall, only two of the participants were given a structured support programme during their first twelve months. When both Gareth and Adam spoke about this support it was evident that both programmes were very much task-focussed and aligned to the SoR Preceptorship Model which is predominantly product driven instead of process driven and rather mechanistic in approach. From listening to Gareth and Adam's perception of the programmes they were offered; it seems as though they both lacked elements of personal and professional development. This would correlate with the SoR model which appears to be limited in terms of professional development and is heavily linked to outcomes. This epistemological approach to the role ignores the process of transformation into the role of a diagnostic radiographer. It has been argued that there is an intricate relationship between knowing and becoming which underpins the students' journey to becoming a new self (Barnett, 2009; Dall'Alba, 2009; Dall'Alba and Barnacle, 2007). Preceptorship schemes need to ensure that there is an ontological aspect to the programme in order to facilitate the process of 'becoming a radiographer.'

The newly qualified radiographers displayed a range of emotions to describe their first few months and as such, support was identified as an integral part of their early experiences. As they began to develop their skills, the graduates look for reassurance and guidance. This may have come in the form of formal support which was structured, allowing them to practice and refine their skills. However, it is evident that 
the uptake in preceptorship in radiography is still relatively low and much of the formal support given to the newly qualified radiographers was task orientated. There were still many of the participants who were not provided with any type of support within their department, despite being newly qualified. Entering an unfamiliar environment left them feeling unvalued and more like a 'number' than a person within the workplace. The influence of Communities of Practice (CoPs) within the workplace means that the graduates had to navigate their way into their new environment in order to become part of the community (Harvey-Lloyd et al., 2019). However, it is recognised that support mechanisms such as structured support, peer support and supportive colleagues are needed to ensure that this happened quickly and successfully.

There are limitations to this study which should be acknowledged at this time. The first is that the study is a qualitative nature and therefore the findings cannot be generalised. However, they are potentially transferable, and this article presents first-hand stories from newly qualified radiographers which may resonate with students, radiographers and educators. It is hoped that these will underpin change and inform practice. The second limitation is the author's previous relationship with the graduates which was a lecturer and course leader. Although it is felt that the close relationship between the researcher and the students was a strength of this study, it could also have been perceived as a limitation. It is difficult to establish if the participants held back information because they did not want the researcher to know exactly how they were feeling. They may not have wanted to say anything negative about their preparation which related to the course, or how they were feeling about the profession. Time was taken to build a new relationship with the participants and with the use of additional data collection tools and the longitudinal design of the study it is hoped that this potential limitation was mitigated as much as possible.

The findings of this study and reflection on the evident lack of formalised support given to newly qualified radiographers, together with the feelings of stress experienced by the graduates would suggest that it is a prudent time to consider how best to support this group of practitioners. It has already been recognised that the needs and expectations of Generation $\mathrm{Z}$ are very different from previous generations, and these were identified as values, perceptions, expectations and motivations in the workforce (Jones et

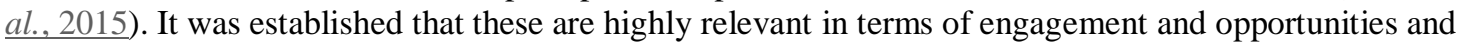
go some way to explain their feelings and emotions during the first few months. It is clear that this generation need a new model of preceptorship which combines the need for skills and competence development with a supportive element which helps graduates to deal with the experience of transition.

This could involve a variety of approaches, utilising peer support, peer assisted learning and coaching (Secomb, 2008; Spouse, 2003; Whitmore, 2002). With preceptorship embracing a coaching approach, the preceptor will develop coaching skills in order to effectively support the newly qualified practitioner and facilitate the development of their potential, whilst maximising their performance (Whitmore, 2002). This collaborative approach which is solution-focused, will encourage newly qualified radiographers to find their own solutions which supports, empowers and raises their self-confidence. Using a workplace coaching approach within this preceptorship model will underpin the development of a culture within departments which facilitates and encourages quality conversations: not only enhancing the personal and professional development of individuals but equipping departments to effectively deal with challenges faced by the NHS (Grant, 2016).

A twelve-month time frame is suggested as this study evidenced that the level of formal support had become less important to the participants as they had grown in confidence and were starting to widen their perspectives and look forward to their future. It was at this point that the participants had become established in their roles and were now well versed in supporting and teaching others.

Below is a proposed preceptorship framework, combining these approaches which is underpinned by the findings of this study (Figure 1). 
Figure 1: A Proposed Preceptorship Framework

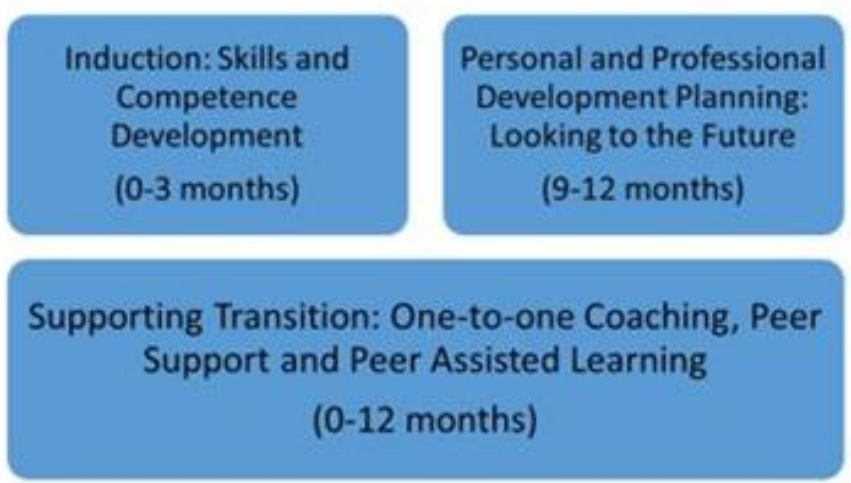

In the literature review, it was suggested that the Flying Start programme introduced in Scotland in 2006 could be considered as a support mechanism for new graduates in England in the future. However, although positively evaluated, it should be emphasised that very few AHPs took part in the programme. Many participants struggled to complete the programme due to time constraints and lack of support. It was also heavily linked to the KSF and consisted of ten units that followed a very structured and generic approach which mainly focussed developing confidence and clinical skills. The proposed framework uses a variety of approaches to support the student and takes into account the individual needs of each newly qualified radiographer. It is suggested that this framework would not only offer the much-needed initial support at this time but also provide a nurturing and stimulating environment in which a structured career pathway for graduates is offered. This also recognises the generation differences in the Generation $\mathrm{Z}$ workforce which the Flying Start programme fails to do.

There are several ways in which HEIs could better prepare radiography students for their transition. Firstly, by ensuring that students are exposed to at least one other department during their degree course. This would mirror the placement experience of other AHP students and give them the opportunity of experiencing more than one imaging department as well as different ways of working. Secondly, the introduction of resilience workshops and transition preparation days which can be delivered and supported by alumni and practice partners. Lastly, consideration should be given to the beginning of preceptorship during their last months in placement in their final year, in order to prepare them more fully for the challenges they will face. This could be developed in partnership with practice and alumni, designed to help the graduates adapt to the move from the supportive environment of the university to one where they were now expected to ensure and prioritise patient throughput, coping with and manging the challenges that this presents is maintained.

\section{Conclusion and Implications for practice}

Preceptorship in diagnostic radiography is still largely underutilised and undervalued by the profession. The SoR preceptorship model discussed in this article needs to be revisited and a new vision of preceptorship presented. Developing a culture of preceptorship and continuing professional development within imaging departments and introducing the right type of preceptorship is essential at these early stages and should not only increase job satisfaction but also overcome staff retention issues within the NHS workforce. The profession still has some way to go in developing its ethos of and application to preceptorship and therefore it is incumbent on Higher Education Institutions, practice partners and alumni to work together to make this a reality, includes preparation for transition to include the development of coaching skills. This should ensure that preceptorship becomes a valued and supported concept within diagnostic radiography, safeguarding the future of the profession. 


\section{Ethical approval}

University of Brighton reference - FREGC-13-010.R1 (23/4/2013), and University Campus Suffolk (former name of the University of Suffolk) (26/4/2013).

\section{ORCID}

Jane Morris

https://orcid.org/0000-0002-0129-9222

Jane Harvey-Lloyd:

https://orcid.org/0000-0001-6706-6790

\section{References}

Banks, P., Roxburgh, M., Kane, H., Lauder, W., Jones, M., Kydd, A., \& Atkinson, J. (2011). Flying Start NHSTM: Easing the transition from student to registered health professional. Journal of Clinical Nursing, 20(23/24), 3567-76. https://doi.org/10.1111/j.1365-2702.2011.03796.x

Barnett, R. (2009). Knowing and becoming in the higher education curriculum. Studies in Higher Education, 34(4), 429-440. https://doi.org/10.1080/03075070902771978

Black, L. L., Jenson, G. M., Mostrom, E., Perkins, J., Ritzline, P. D., Hayward, L., \& Blackmer, B. (2010). The first year of practice: an investigation of the professional learning and development of promising novice physical therapists. Physical Therapy, 90(12), 1758-1773. https://doi.org/10.2522/ptj.20100078

Bligh, J. G. (1992). Independent learning among general practice trainees: an initial survey. Medical Education, 26: 497-502. https://doi.org/10.1111/j.1365-2923.1992.tb00212.x

Boychuk Duchscher, J. B. (2008). A Process of Becoming: The Stages of New Nursing Graduate Professional Role Transition. The Journal of Continuing Education in Nursing, 39(10), 451-452. https://doi.org/10.3928/00220124-20081001-03

Braun, V., \& Clark, V. (2006). Using a thematic analysis in psychology. Qualitative Research in Psychology, 3(2), 77-101. https://doi.org/10.1191/1478088706qp063oa

Cohen, L., Manion, L., \& Morrison, K. (2000). Research methods in education (5th ed.). RoutledgeFalmer.

Cole, M., John-Steiner, V., Scribner, S., \& Souberman, E. (Eds.). (1978). Mind in society: The development of higher psychological processes. L. S. Vygotsky. Harvard U Press.

Cresswell, J. W. (2007). Qualitative inquiry and research design. Choosing among five approaches (2nd ed.). Sage.

Dall'Alba, G. (2009). Learning professional ways of being: Ambiguities of becoming. Educational Philosophy and Theory, 14(1), 34-45. https://doi.org/10.1111/j.1469-5812.2008.00475.x

Dall'Alba, G., \& Barnacle, R. (2007). An ontological turn for higher education. Studies in Higher Education, 32(6), 679-691. https://doi.org/10.1080/03075070701685130

Decker, S. (2009). The lived experience of newly qualified radiographers (1950-1985): An oral history of radiography. Radiography, 15(1), 72-77. https://doi.org/10.1016/j.radi.2009.09.009

Department of Health (DoH). (2010). Preceptorship framework for newly qualified nurses, midwives and allied health professionals. London. https://www.networks.nhs.uk/nhs-networks/ahpnetworks/documents/dh_114116.pdf

Fenwick, J., Hammond, A., Raymond, J., Smith, R., Gray, J., Foureur, M., Homer, C., \& Symon, A. (2012). Surviving not thriving: a qualitative study of newly qualified midwives experiences of their transition to practice. Journal of Clinical Nursing, 21(13-14), 2054-63.

Fleer, M. (1990). Scaffolding conceptual change in early childhood. Research in Science Education, 20(1), 114-123. https://doi.org/10.1007/BF02620486

Gerrish, C. (1990). Fumbling along. Nursing Times, 86(30), 35-37.

Grant, A. (2016). The third 'generation' of workplace coaching: creating a culture of quality conversations. Coaching: an international journal of theory, research and practice, 10(1), 3753. https://doi.org/10.1080/17521882.2016.1266005

Harvey-Lloyd, J. (2018). Being and becoming a diagnostic radiographer [Doctoral dissertation, University of Brighton.]. University of Brighton Research Portal. https://research.brighton.ac.uk/en/studentTheses/being-and-becoming-a-daignostic-radiographer 
Harvey-Lloyd, J. M., Morris, J., \& Stew, G. (2019). Being a newly qualified diagnostic radiographer: Learning to fly in the face of reality. Radiography, 25(3), 63-67. https://doi.org/10.1016/j.radi.2019.01.007.

Harvey-Lloyd, J. M., Stew, G. \& Morris, J. M. (2012). Role transition: From student to practitioner. Synergy: Imaging and Therapy Practice, 2012(June), 9-14.

Jones, K., Warren, A., \& Davies, A. - Health Education England (2015). Mind the Gap. Exploring the needs of early career nurses, and midwives in the workplace. Summary report from Birmingham and Solihull Local Education and Training Council Every Student Counts Project. London: Health Education England. https://www.nhsemployers.org//media/Employers/Documents/Plan/Mind-the-Gap-Smaller.pdf

Kafle, N. P. (2011). Hermeneutic phenomenological research method simplified. Bodhi: An Interdisciplinary Journal, 5(1), 181-200. https://doi.org/10.3126/bodhi.v5i1.8053

Langdridge, D. (2007). Phenomenological Psychology: Theory, research and method. Pearson Education. Leavy, P. (2009). Method meets art: Art-based research practice. The Guildford Press.

MacKay, S. J., Anderson, A. C., \& Hogg, P. (2008). Preparedness for clinical practice - perceptions of graduates and their work supervisors. Radiography, 14(3), 226-32. https://doi.org/10.1016/j.radi.2007.05.001

McAllister, M. \& McKinnon, J. (2009). The importance of teaching and learning in the health disciplines: A critical review of the literature. Nurse Education Today, 29(4) 371-379. https://doi.org/10.1016/j.nedt.2008.10.011

Mooney, M. (2007). Facing registration; the expectations and the unexpected. Nurse Education Today, 27(8), 840-847. https://doi.org/10.1016/j.nedt.2006.11.003

Naylor, S., Ferris, C., \& Buton, M. (2016). Exploring the transition from student to practitioner in diagnostic radiography. Radiography 22(2), 131-136. https://doi.org/10.1016/j.radi.2015.09.006

NHS Confederation. (2014). The 2015 Challenge Declaration. NHS Confederation. https://www.nhsconfed.org/supporting-members/influence/2015-challenge/2015-challengedeclaration

NHS Education for Scotland. (2006). Flying start NHS. https://learn.nes.nhs.scot/735/flying-start-nhs

Pringle, J., Drummond, J., McLafferty, E., \& Hendry, C. (2011). Interpretative phenomenological analysis: a discussion and critique. Nurse Researcher 18(3), 20-24. https://doi.org/10.7748/nr2011.04.18.3.20.c8459

Richez, M. (2014). Resilience-building strategies for nurses in transition. Journal of Continuing Education in Nursing, 45(2), 54-55. https://doi.org/10.3928/00220124-20140124-12

Scholes, J. (2008). Why health care needs resilient practitioners. Nursing in Critical Care, 13(6), 281-5. https://doi.org/10.1111/j.1478-5153.2008.00306.x

Secomb, J. (2008). A systematic review of peer teaching and learning in clinical education. Journal of Clinical Nursing, 17(6), 703-716. https://doi.org/10.1111/j.1365-2702.2007.01954.x

Smith, R. A., \& Pilling, S. (2007). Allied health program - supporting the transition from student to professional in an interdisciplinary program. Journal of Interprofessional Care, 21(3), 265-276. https://doi.org/10.1080/13561820701259116

Spouse, J. (2003). Professional learning in nursing. Blackwell Science. https://doi.org/10.1002/9780470774496

Tryssenaar, J., \& Perkins, J. (2001). From student to therapist: Exploring the first year of practice. The American Journal of Occupational Therapy, 55, 19-27. https://doi.org/10.5014/ajot.55.1.19

Whitmore, J. (2002). Coaching for performance (3rd ed.). Nicholas Brealey Publishing.

Wiercinski, A. (2009). Hans-Georg Gadamer and the truth of hermeneutic experience. Analecta Hermeneutica, 1, 3-14. https://philpapers.org/rec/WIEHGA 